Neurosurg Focus 17 (6):E11, 2004

\title{
Cervical osteomyelitis: a brief review
}

\author{
Bryan Barnes, M.D., Joseph T. Alexander, M.D., and Charles L. Branch JR., M.D. \\ Department of Neurosurgery, Wake Forest University Health Sciences, Winston-Salem, \\ North Carolina
}

\begin{abstract}
Object. The authors conducted a literature-based review of the etiology, diagnosis, and treatment of cervical vertebral osteomyelitis (CVO).

Methods. A Medline (PubMed) search using the key words "cervical vertebral osteomyelitis" yielded 256 articles. These were further screened for relevance, yielding 15 articles. Each publication was reviewed, and several others not identified in the PubMed search were screened and included in the review according to relevance. Each article was identified as involving either the epidemiology/etiology, diagnosis, or treatment of CVO. Separate categories were created for case reports and general reviews.

Conclusions. Cervical vertebral osteomyelitis has a spectrum of origins, which include spontaneous, postoperative, traumatic, and hematogenously spread causes. The majority of patients have medical risk factors and comorbidities that include diabetes, trauma, drug abuse, and infectious processes in extraspinal areas. The diagnosis of CVO can be accomplished in most cases by using plain x-ray films and computerized tomography scans. Nevertheless, preferential use of magnetic resonance imaging in cases in which there is a neurological deficit is helpful in identifying epidural compressive processes. Treatment for CVO can be successfully initiated with intravenous antibiotic therapy. Nevertheless, in cases in which there is a neurological deficit, spinal deformity and/or progressive lysis, or intractable pain, the earliest feasible surgical intervention with debridement and fusion is warranted.
\end{abstract}

KEY WoRdS • cervical spine • osteomyelitis - spinal infection

Surgical experience with spinal osteomyelitis dates to the early 20th century, when Hibbs ${ }^{5}$ performed wiring of the lumbar spinous processes to treat Pott disease, or tuberculosis of the spine. In subsequent decades, spinal surgery techniques that had been developed to treat spinal osteomyelitis were expanded to treat degenerative spine disease. Degenerative spine disease and acute trauma now represent a majority of the spine surgeon's practice. Spinal osteomyelitis still represents a less frequently encountered but nevertheless difficult challenge for the spine surgeon. Cervical osteomyelitis in particular can have potentially catastrophic implications for the patient if not diagnosed and treated early. Based on our review of the available literature on cervical osteomyelitis, we discuss the key elements of diagnosis and management of the disease.

\section{CLINICAL MATERIAL AND METHODS}

A Medline (PubMed) search using the key words "cervical vertebral osteomyelitis" yielded 256 articles, which were screened for further relevance. Fifteen relevant articles were found, and these were classified according to their emphasis on the epidemiology/etiology, diagnosis, or treatment of CVO. A separate category was created for general reviews and case reports. Articles were then thor-

Abbreviations used in this paper: $\mathrm{CVO}=$ cervical vertebral osteomyelitis; $\mathrm{MR}=$ magnetic resonance. oughly reviewed for content. Data were subclassified into epidemiological, diagnostic, and treatment data. In cases in which general reviews were presented, data pertinent to each of the three categories were subdivided accordingly. Case reports were noted but not included in the data analysis. Several additional articles were identified based on citations in papers identified through the Medline (PubMed) search.

\section{RESULTS}

\section{Epidemiology/Etiology}

Four comprehensive series of patients with CVO were found. ${ }^{1,3,7,10}$ In three of the four reports, cases of CVO were extrapolated from a series of patients with spinal vertebral osteomyelitis and distinguished from cases of thoracic and lumbosacral osteomyelitis. Reports of the percentage of cases of spinal osteomyelitis that exclusively involved the cervical spine ranged from 3 to $11 \%$. The mean age of the patients with CVO ranged from 57 to 60 years. A majority of patients in all series were men, with a range of 66 to $87 \%$ for male sex. Medical comorbidities or serious medical illnesses were identified in many of the patients (range 43-64\%), but spontaneous cases of osteomyelitis were also reported. ${ }^{1,13}$ Major illnesses and comorbidities that were identified included alcoholism, dental work, liver disease, diabetes, previous surgery/discography, drug abuse, renal insufficiency, heart disease, tuberculosis, and 
distant focus of infection. ${ }^{1-4,7,8,10}$ The infectious organism that was most frequently identified was Staphylococcus aureus (25-64\% of cases), followed by Streptococcus spp. $(8-12 \%)$. Estimates of negative results on biopsy samples or intraoperative cultures ranged from 31 to $42 \%$.

\section{Time to Diagnosis}

Three publications provided data on the mean time between onset of symptoms and diagnosis; the range was between 14.4 and 17.9 weeks. Symptoms included neck pain in 71 to $100 \%$ of patients with CVO. Only one account of the prevalence of febrile symptoms among patients was provided; the value given was $66 \%$. Stratification of diagnostic imaging was provided in the same publication ${ }^{1}$ as follows: $55 \%$ of patients were diagnosed based on plain xray films and MR imaging, 25\% on plain x-ray films and myelographic studies, and $20 \%$ on CT scans and plain Xray films. The authors of this publication noted, however, that the diagnosis of patients based on myelography predated the availability of MR imaging in their institution.

The prevalence of paravertebral or epidural abscess was estimated to be $53 \%$ in one series (Figs. 1 and 2). ${ }^{1} \mathrm{Be}-$ tween 44 and $59 \%$ of patients with CVO suffered from a neurological deficit at the time of diagnosis; there was a high correlation of neurological deficit to epidural abscess. ${ }^{1,3,7,10}$ Only one paper ${ }^{1}$ tabulated distribution of the level of involvement: C5-6 (40\%) followed by C6-7 (20\%). Isolated cases of upper cervical (that is, C-1 and C-2) infections are reported. ${ }^{6,14}$

\section{Treatment Modalities}

Surgical Management. All identified series of CVO were strongly biased toward surgical intervention; two of these series were based on the authors' surgical experi-

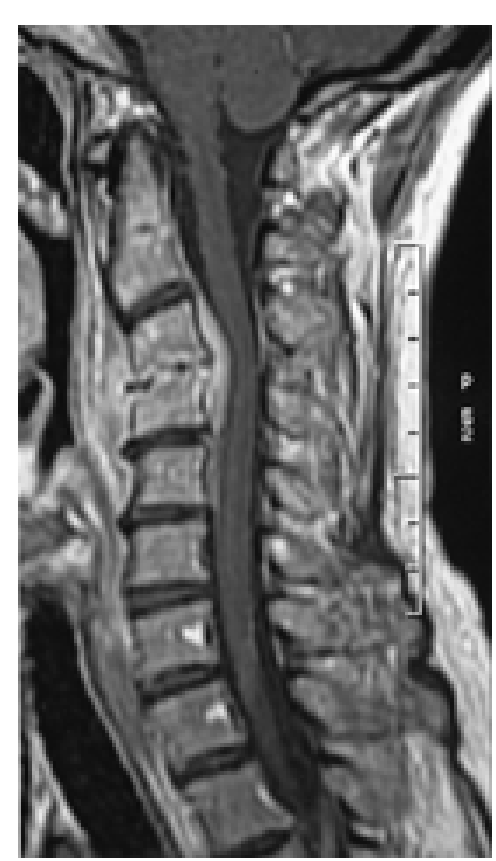

Fig. 1. Sagittal $\mathrm{T}_{1}$-weighted MR image demonstrating cervical vertebral osteomyelitis at C3-4. Note the hyperintense epidural abscess.

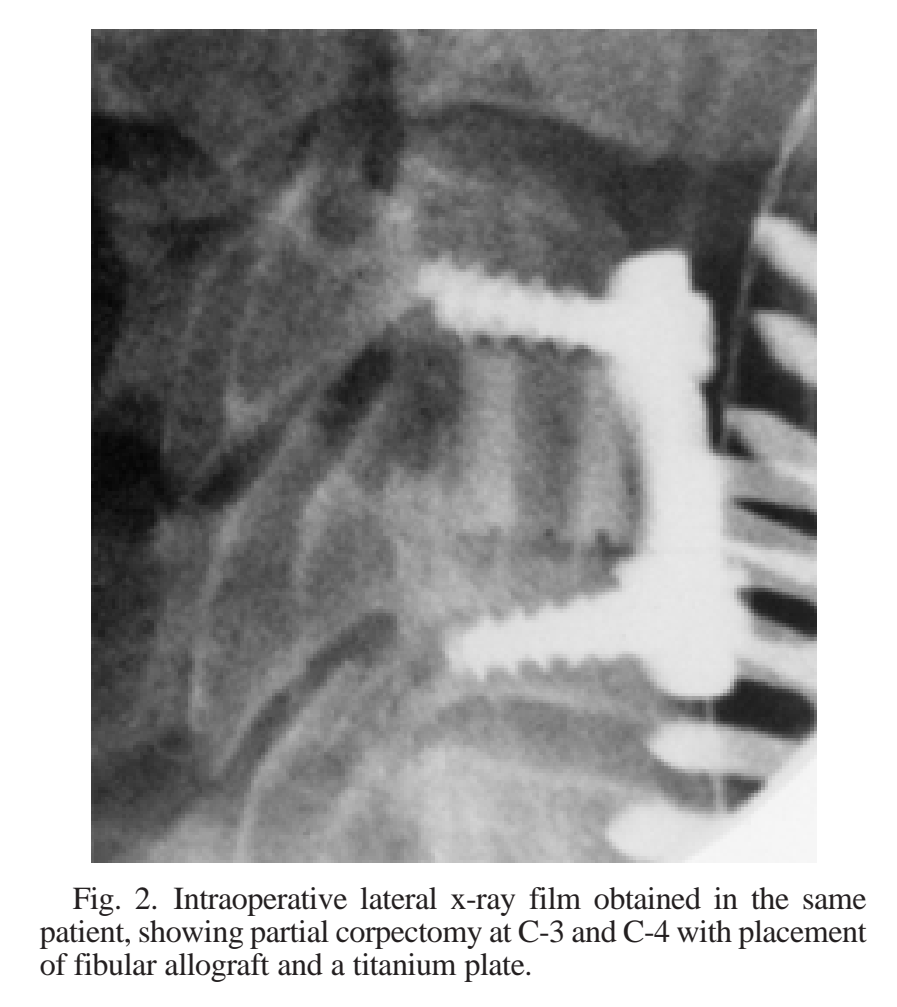

ence only, and no estimates of the percentage of nonsurgically treated cases were provided..$^{3,9,10}$ There were two estimates of the rate of surgical intervention in all cases of spinal osteomyelitis, with the number of patients requiring such intervention ranging from 18 to $43 \%,{ }^{1,7}$ Nevertheless, these percentages were not stratified for cervical compared with noncervical disease.

Nonsurgical Management. Indications for nonsurgical management included absence of neurological deficit, good pain control with analgesic medications, no associated epidural abscess, and no significant deformity. $3,7,9,10$ Treatment duration and type of antibiotic therapy are dictated by the infecting organism; with a predominance of $S$. aureus and other Gram-positive organisms, vancomycin and the cephalosporins are frequently administered for long-term intravenous therapy.

Additional Strategies. For those articles in which the surgical procedure was specified, direct anterior debridement was always the approach of choice..$^{1,3,7,9-12}$ After anterior debridement, several additional surgical strategies were used. Options exercised immediately after anterior debridement included the following: 1) no further treatment; 2) anterior fusion alone; and 3 ) anterior and posterior fusion. In some cases, surgeons elected to perform a delayed anterior fusion, specifically when gross purulence was encountered. In one of the surgical series, the authors advocated a delayed posterior cervical stabilization after anterior debridement and fusion. ${ }^{3}$

\section{Treatment Outcomes}

Patients with CVO who underwent surgery generally had favorable outcomes, with improvement or return to normal neurological function occurring in 66 to $83 \%$ of those who underwent anterior debridement. ${ }^{7,10}$ Multi- 
variate analysis of outcomes for patients with all types of spinal osteomyelitis showed that independent risk factors for poor outcome included longer time to diagnosis, neurological impairment, and hospital-acquired infections; no stratification was made, however, for cervical compared with noncervical cases. ${ }^{10}$ Interestingly, the severity of a medical comorbidity was not an independent predictor of poor outcome. ${ }^{10}$

Relapse was encountered in $14 \%$ of cases of spinal osteomyelitis; persistent pain was usually the main symptom. Statistically significant predictors of relapse included recurrent bacteremia, a chronically draining sinus, and a paravertebral abscess. ${ }^{10}$

\section{DISCUSSION}

One of the most insidious aspects of CVO is the lengthy period between onset of symptoms (predominantly neck pain) and diagnosis. It seems reasonable to infer that the delay in diagnosis is probably correlated with the approximately $50 \%$ incidence of neurological deficit in patients presenting with $\mathrm{CVO}$ that is described in the literature. Although investigators in many series report the use of plain x-ray films with computerized tomography scanning or myelography to diagnose $\mathrm{CVO}$, the most reasonable approach with currently available imaging technology would seem to be plain x-ray films followed by MR imaging to detect an epidural abscess.

Despite the high frequency of delayed diagnosis, timely intervention with surgery and/or medical therapy can be extremely efficacious. Indications for surgery include intractable pain, progressive vertebral body destruction and/ or kyphotic deformity, presence of a neurological deficit, and presence of an epidural abscess. Surgery is noted by most authors to provide an excellent prognosis overall; in particular it will improve neurological function in a majority of cases.

Surgeons universally chose an anterior cervical approach for initial debridement. Various strategies have been successful after debridement, including no fusion, fusion at the time of surgery, delayed anterior fusion, and anterior debridement and fusion followed by posterior stabilization. Although it is well known that fusion at the time of debridement is a viable strategy, the presence of gross purulence at the surgical site may give the surgeon pause. As long as the patient has the proper activity restrictions and orthosis, delaying fusion in the presence of purulence seems reasonable. Nevertheless, the use of interbody grafting for fusion at some point seems prudent, given that most patients with osteomyelitis will have an increased risk of deformity. Persistent infection at the site of the implants is extremely rare, but revision surgery and repeated debridement can successfully treat such infections if they occur. ${ }^{11}$

Excellent outcomes after surgery for CVO were noted in this review. These outcomes included an improvement in neurological deficit (when present initially). Spinal ar- throdesis was also successful in the overwhelming majority of cases.

\section{CONCLUSIONS}

Overall, CVO is an entity that is often not recognized in a timely fashion. Prompt surgical evaluation and treatment are warranted, in conjunction with medical management for specific indications. The prognosis is generally good when surgical therapy is initiated.

\section{References}

1. Belzunegui J, Del Val N, Intxausti JJ, et al: Vertebral osteomyelitis in northern Spain. Report of 62 cases. Clin Exp Rheumatol 17:447-452, 1999

2. D'Elia JA, Cooppan R, Younger D, et al: Cervical osteomyelitis. JAMA 241:29, 1979

3. Dimar JR, Carreon LY, Glassman SD, et al: Treatment of pyogenic vertebral osteomyelitis by anterior debridement and fusion followed by delayed posterior spinal fusion. Spine 29: 326-332, 2004

4. Ghanayem AJ, Zdeblick TA: Cervical spine infections. Orthop Clin North Am 27:53-67, 1996

5. Hibbs RA: An operation for progressive spinal deformities. NY Med J 93:1013-1016, 1911 (Reference unverified)

6. Lam CH, Either R, Pokrupa R: Conservative therapy of atlantoaxial osteomyelitis. A case report. Spine 21:1820-1823, 1996

7. McHenry MC, Easley KA, Locker GA: Vertebral osteomyelitis: long-term outcome for 253 patients from 7 Clevelandarea hospitals. Clin Infect Dis 34:1342-1350, 2002

8. Pinckney LE, Currarino G, Highenboten CL: Osteomyelitis of the cervical spine following dental extraction. Radiology 135: 335-337, 1980

9. Rezai AR, Woo HH, Errico TJ, et al: Contemporary management of spinal osteomyelitis. Neurosurgery 44:1018-1026, 1999

10. Schimmer RC, Jeanneret C, Nunley PD, et al: Osteomyelitis of the cervical spine: a potentially dramatic disease. J Spinal Disord Tech 15:110-117, 2002

11. Shad A, Shariff S, Fairbank J, et al: Internal fixation for osteomyelitis of the cervical spine: the issue of persistence of culture positive infection around the implants. Acta Neurochir 145: 957-960, 2003

12. Stone JL, Cybulski GR, Rodriguez J, et al: Anterior cervical debridement and strut-grafting for osteomyelitis of the cervical spine. J Neurosurg 70:879-883, 1989

13. von Eckardstein K, Spuler A, Brauer C, et al: Spontaneous cervical osteomyelitis due to Yersinia enterocolitica in a nonimmunocompromised host. Eur J Microbiol Infect Dis 23: 66-68, 2004

14. Wiedau-Pazos M, Curio G, Grusser C: Epidural abscess of the cervical spine with osteomyelitis of the odontoid process. Spine 24:133-136, 1999

Manuscript received October 15, 2004

Accepted in final form November 4, 2004.

Address reprint requests to: Bryan Barnes, M.D., Department of Neurosurgery, Wake Forest University, Medical Center Boulevard, Winston-Salem, North Carolina 27157-1029. email: bbarnes @wfubmc.edu. 